\title{
Long-term effect of prazosin and losartan administration on blood pressure, heart, carotid artery, and acetylcholine induced dilation of cardiovascular system of young Wistar rats and SHR
}

\author{
Frantisek Kristek, Magdalena Malekova and Sona Cacanyiova \\ Institute of Normal and Pathological Physiology, Centre of excellence for examination of regulatory role of nitric oxide in \\ civilisation diseases, Slovak Academy of Sciences, Sienkiewiczova 1, 81371 Bratislava, Slovak Republic
}

\begin{abstract}
The long-term effects of prazosin and losartan administration on blood pressure, trophicity of the heart and carotid arteries, and responses of the cardiovascular system to acetylcholine, were studied in Wistar rats and spontaneously hypertensive rats (SHRs). Four-week-old rats were treated with prazosin (10 mg/kg b.w./day in tap water) or losartan $(20 \mathrm{mg} / \mathrm{kg}$ b.w./day in tap water) for 5-6 weeks. BP was measured by plethysmographic method. Ten animals of each group were subjected to in vivo studies and subsequent to morphological investigations. The right jugular vein was cannulated for administration of acetylcholine $(0.1,1$, and $10 \mu \mathrm{g})$. After perfusion with a glutaraldehyde fixative $(120 \mathrm{mmHg})$, the carotid arteries were embedded in Durcupan ACM, and the inner diameter (ID), wall thickness (WT) (tunica intima and media), cross sectional area (CSA) (tunica intima and media), and WT/ID ratio were calculated. In Wistar rats and SHRs, prazosin and losartan administration produced a decrease in the blood pressure and trophicity of the heart. In Wistar rats, both drugs decreased the WT, CSA, and the WT/ID ratio. In addition, these drugs increased the circumferential stress of the artery without affecting the ID. In contrast, in the SHRs, only losartan administration produced these effects. Importantly, both the drugs improved the responses to acetylcholine in SHRs.
\end{abstract}

Key words: Carotid artery - Prazosin — Losartan - Structure - Acetylcholine responses

Abbreviations: Ang II, angiotenzín II; BP, blood pressure; CSA, cross sectional area; HW, heart weight; ID, inner diameter; SHR, spontaneously hypertensive rats; WT, wall thickness.

\section{Introduction}

Spontaneously hypertensive rats (SHRs) are frequently used as animal models to document the functional and structural alterations in the cardiovascular system that are induced by hypertension. In the first few weeks after birth, there is a rapid increase in the blood pressure (BP) in SHRs and normotensive Wistar rats. However, a majority of the experiments, including those described in the original study by Okamoto and Aoki (1963) have reported that until approximately 4 weeks after birth, there is no difference in BP

Correspondence to: Frantisek Kristek, Institute of Normal and Pathological Physiology, Slovak Academy of Sciences, Sienkiewiczova 1,813 71 Bratislava, Slovak Republic

E-mail: Frantisek.Kristek@savba.sk between SHRs and age-matched Wistar rats. However, from the $4^{\text {th }}$ to $9^{\text {th }}$ postnatal week, the increase in BP in addition to hypertrophy of the heart and arterial wall progresses more rapidly in SHRs compared to Wistar rats. Further, during the $9^{\text {th }}$ postnatal week, there is already a significant increase in BP and hypertrophy of the heart and arterial walls of the conduit and resistant arteries. In the later periods, these alterations continue to progress; however, the dynamics of these changes is slower (Cebova and Kristek 2011). Since the increase in BP and structural alterations in the cardiovascular system begin approximately during the same ontogenetic period, the literature findings do not consistently define which of these effects provides the signal to trigger subsequent pathological changes (Gray 1984; Clubb et al. 1987; Head 1989; Cebova and Kristek 2011). Nevertheless, it appears that the rapid increase in BP is the 
most important risk factor, which triggers the functional and structural alterations that are observed in cardiovascular diseases. When the alterations are induced early during ontogeny, it can have important consequences, which could persist till adulthood. Experimental studies have shown that preventing these pathological alterations is more effective when the intervention is initiated before the changes are fully developed and stabilized.

Several regulatory systems are involved in maintaining the optimal functions of the cardiovascular system. Among the most important systems are the sympathetic nervous system (SNS) and the renin-angiotensin system (RAS). Both of these systems are tightly linked, and therefore, any imbalance between them has a serious impact on BP and the function and structure of the heart and vascular wall, which results in an inadequate supply of the blood to the individual organs. Modulation of sympathetic activity by angiotensin II (ang II) (at the pre- and postsynaptic level) and complete suppression of the facilitatory role of ang II on noradrenaline release after chronic treatment with losartan further substantiates the close relationship between these two systems (Ruiz-Gayo et al. 2000). The arteries in SHRs receive a greater density of sympathetic innervations compared to blood vessels in normotensive strains (Ruiz-Gayo et al. 2000). The SNS plays a crucial role in the pathogenesis of hypertension because, in addition to increasing the resting sympathetic tone, it induces cardiovascular hypertrophy (Adams et al. 1989). The majority of the effects of SNS on the cardiovascular system are mediated via the alfal adrenoceptors. The RAS exerts potent vasoconstrictor effects via ang II, and it is directly involved in the vascular (Griendling and Alexander 1995) and cardiac (Crawford et al. 1994) remodeling that are observed in response to chronic and acute hypertension. The majority of the ang II effects in the cardiovascular system are mediated via the AT1 receptors.

The aim of this study is to characterize the influence of prazosin ( $\alpha 1$ antagonist) and losartan (AT1 receptor antagonist) on the cardiovascular system of Wistar rats and SHRs during the phase when the increases in BP are further accelerated. These influences appear to coincide with the period during which there is cardiac and vascular hypertrophy. Interference with the key points of the regulatory systems, particularly early during ontogeny, provides a good opportunity to prevent the development of pathological alterations.

\section{Materials and Methods}

During the experiments, the animals were housed at room temperature $\left(22-24^{\circ} \mathrm{C}\right)$ with a $12 \mathrm{~h}$ light/dark cycle and fed with a regular pellet diet. All of the procedures were performed in accordance with the guidelines indicated in the 'Guide for the Use of Laboratory Animals' (Ethics Committee for Experimental Work, Slovak Academy of Sciences, 1995).

Four-week-old Wistar rats were used in this study. The animals were divided into six groups of 10 animals each: 1) Wistar rats; 2) prazosin-treated Wistar rats; 3) losartantreated Wistar rats; 4) SHRs; 5) prazosin-treated SHRs; and 6) losartan-treated SHRs. Prazosin (10 mg/kg b.w./day) and losartan (20 mg/kg b.w./day) were dissolved in drinking water and were administered by gavage. The experiment lasted for 5-6 weeks. The BP was measured noninvasively by using the plethysmographic method on the tail artery in pre-warmed animals from each of the groups every week.

After 5-6 weeks of treatment, the animals from each group were subjected to in vivo investigation and were subsequently utilized for morphological examination.

\section{In vivo study}

The animals were anesthetized using ketamine $(0.25 \mathrm{ml} /$ $100 \mathrm{~g}$ b.w.) and xylazine (0.1 ml/100 g b.w.) (Zentiva, Czech Republic), which were administered i.p. The right jugular vein was cannulated for administering the respective drugs. Heparin (25 i.u.) was injected into the jugular vein immediately after preparation. The right carotid artery was cannulated and connected to a pressure transducer. Mean arterial pressure was recorded with a Physioscript Schwarzer. After stabilization (approximately $10 \mathrm{~min}$ ), acetylcholine (Zentiva, Czech Republic) was administered via the jugular vein (at doses $0.1,1$ and $10 \mu \mathrm{g}$ ). The drugs were administered in a random order. Individual stimuli were applied in 10-15 min intervals after the BP returned to the basal level and remained stable.

\section{Morphological analysis}

After the in vivo functional analysis, the animals were sacrificed by anesthetic overdose. Their chest was opened, and the cardiovascular system was perfused with a fixative (300 mmol/l glutaraldehyde in $100 \mathrm{mmol} / \mathrm{l}$ phosphate buffer) at a constant pressure of $120 \mathrm{mmHg}$ for $10 \mathrm{~min}$ via a cannula placed in the left ventricle. After the perfusion was performed, the heart was excised and weighed. The middle section of the carotid artery was excised, cleaned, and divided into three segments (approximately $1 \mathrm{~mm}$ long) and were embedded in Durcupan ACM. Two randomly selected pieces of the artery were cut perpendicular to the longitudinal axis, and the inner circumference and arterial wall thickness (tunica intima and tunica media) were measured using light microscopy. The inner diameter and cross sectional area (including the tunica intima 


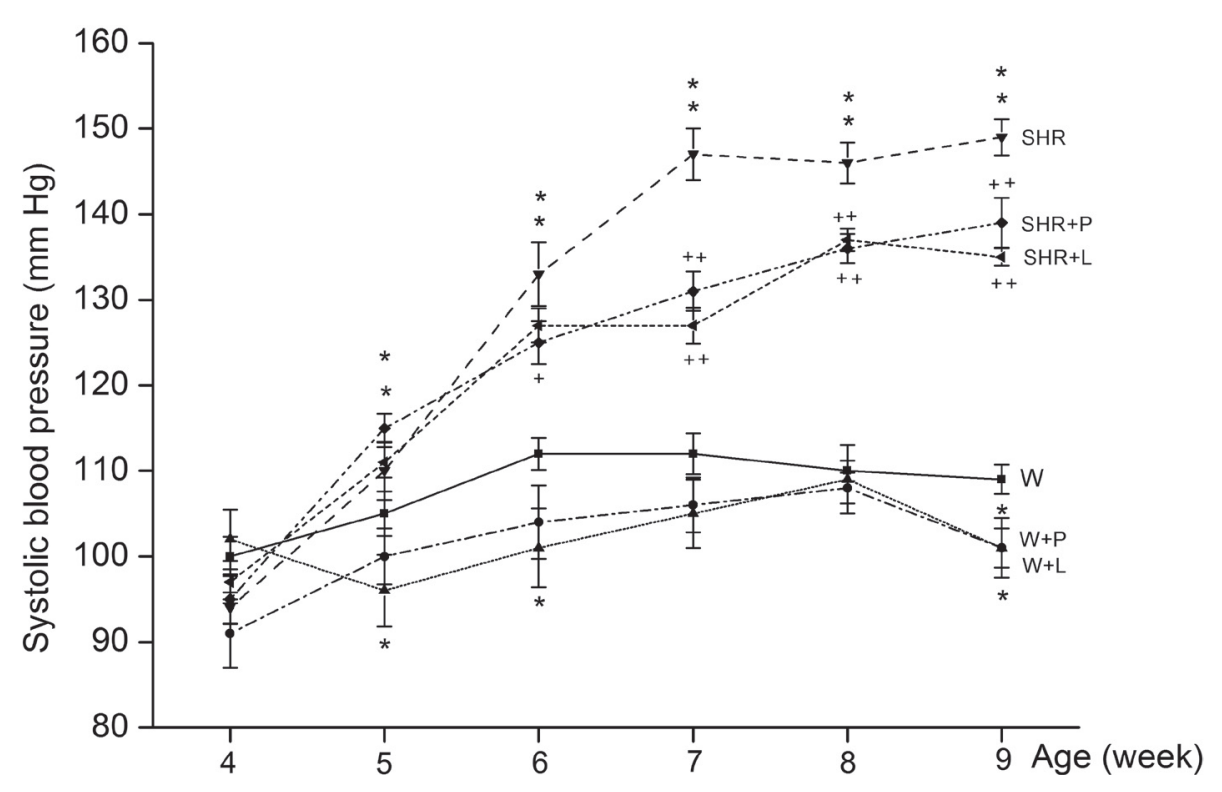

Figure 1. Systolic blood pressure of Wistar rats (W), Wistar rats treated with prazosin $(\mathrm{W}+\mathrm{P})$, Wistar rats treated with losartan $(\mathrm{W}+\mathrm{L})$, spontaneously hypertensive rats (SHR), SHR receiving prazosin $(\mathrm{SHR}+\mathrm{P})$ and SHR receiving losartan $(\mathrm{SHR}+\mathrm{L})$. The values represent the data at the end of each week from 4 to 9 weeks of age. Data are means \pm S.E.M. ${ }^{\star} p<0.05,{ }^{* *} p<0.01 v s$. Wistar rats; ${ }^{+} p<0.05,{ }^{++} p<0.01$ vs. SHR.

and tunica media) were calculated (for details see Kristek 2000).

The data obtained are expressed as the mean \pm S.E.M. The statistical significance was assessed by using ANOVA and Bonferroni test for unpaired variables. Results were considered to be statistically significant when $p<0.05$.

\section{Results}

\section{General cardiovascular parameters}

At the beginning of the experiment, the BP did not differ significantly between the groups. However, from the $4^{\text {th }}$ week after-birth, the BP in the SHR group increased rapidly and was significantly increased in comparison to the Wistar rat group during and after the $5^{\text {th }}$ week. The administration of prazosin and losartan to the Wistar rats and SHRs produced a slight, albeit significant, slowing of this BP increase. At the end of the experiment, there was an increase in the BP of the SHR group compared to the Wistar group (Fig. 1).

There was no difference in the heart weight (HW) (after perfusion of the whole body with fixative) between the Wistar rats and the SHRs. Administration of prazosin and losartan significantly decreased the HW in the Wistar rats and SHRs. Due to the lower body weight of the SHRs, the ratio of heart weight/body weight (HW/BW) in the SHRs was increased in comparison to the Wistar rats. Administration of prazosin and losartan resulted in a pronounced decrease in the HW/BW ratio in the Wistar rats and the SHRs (Fig. 2).

\section{Geometry of the carotid artery}

The wall thickness (WT) of the carotid artery was increased in the SHRs compared to the Wistar rats. The treatment of the Wistar rats with prazosin and losartan produced a decrease in the WT. In the SHRs, a decrease in the WT was observed only in response to losartan administration (Fig. 3A).

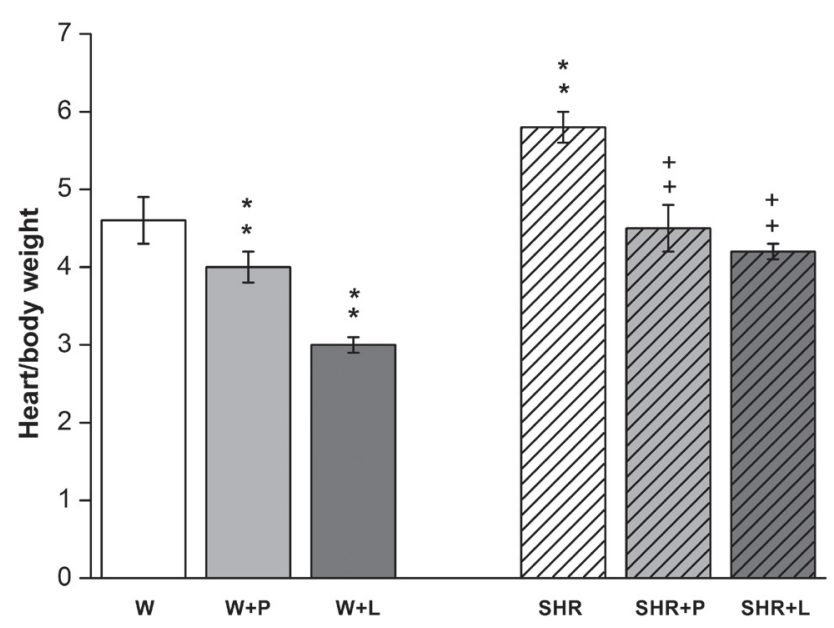

Figure 2. Heart/body weight ratio (mg/g) of control Wistar rats, Wistar rats treated with prazosin, Wistar rats treated with losartan, spontaneously hypertensive rats, SHR receiving prazosin, and SHR receiving losartan. The values represent the data at the end of the experiment. Data are means \pm S.E.M. ${ }^{\star *} p<0.01 v s$. Wistar rats; ${ }^{++} p<0.01 v s$. SHR. For abbreviations see Fig. 1. 

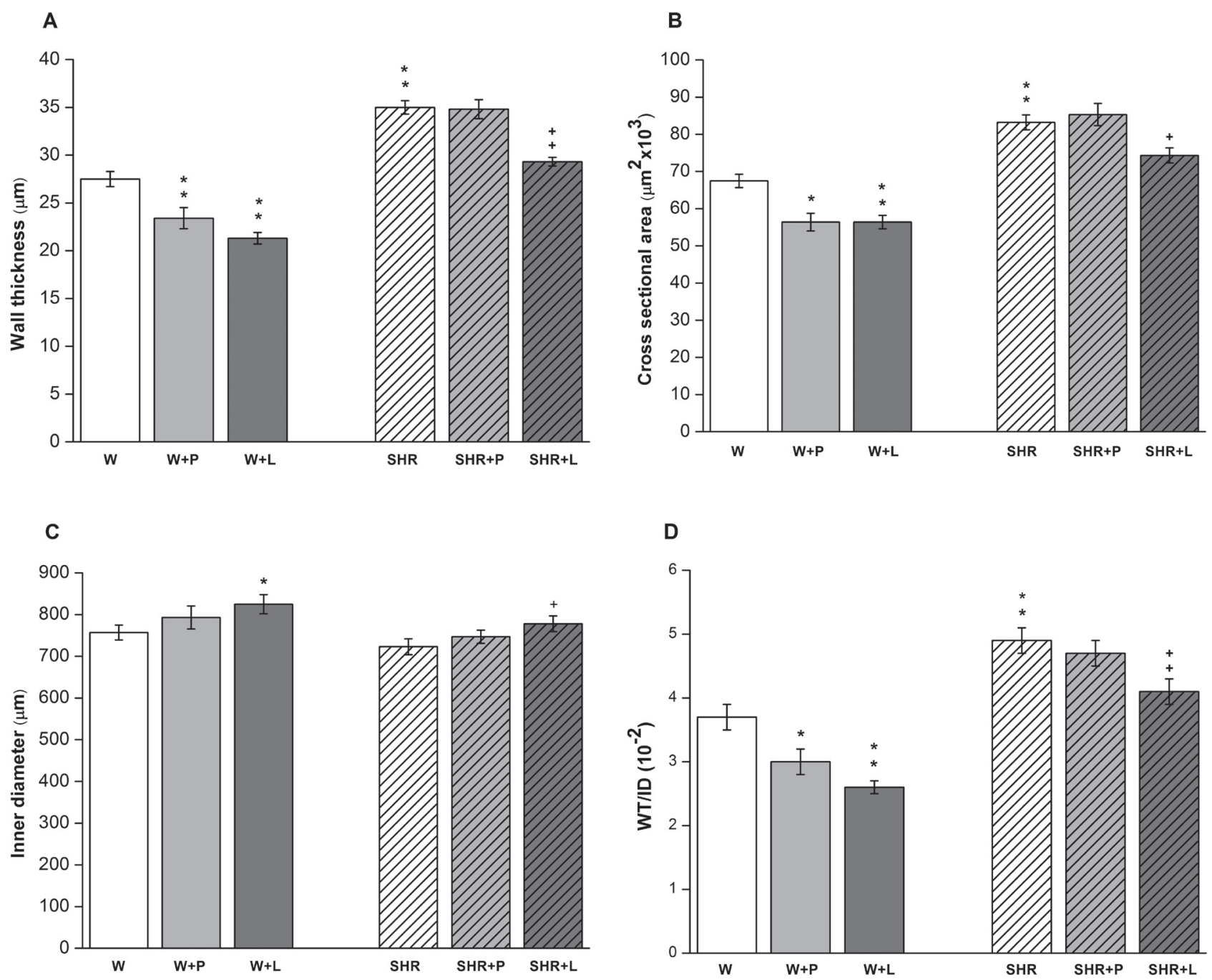

Figure 3. Wall thickness (A), cross sectional area (B), inner diameter (C), and WT/ID ratio (D) of control Wistar rats, Wistar rats treated with prazosin, Wistar rats treated with losartan, spontaneously hypertensive rats, SHR receiving prazosin, and SHR receiving losartan. The values represent the data at the end of the experiment. Data are means \pm S.E.M. ${ }^{\star} p<0.05,{ }^{\star *} p<0.01 v s$. Wistar rats; ${ }^{+} p<0.05$, ${ }^{++} p<0.01 v s$. SHR. For abbreviations see Fig. 1.

The cross-sectional area (CSA) of the carotid artery of the SHRs was increased in comparison to the normotensive Wistar rats. The administration of prazosin and losartan to the Wistar rats elicited a significant decrease in the arterial wall mass, whereas in the SHRs, these effects were noted only with losartan administration. In the prazosin-treated SHRs, there was no effect on the CSA (Fig. 3B).

Inner diameter (ID) of the carotid artery did not differ between the Wistar rats and the SHRs. No effect on the ID was observed in the Wistar rats and the SHRs after prazosin administration; however, there was a mild, yet significant, increase in the ID after losartan administration in the Wistar rats and the SHRs (Fig. 3C).
Ratio WT/ID of the carotid artery was increased in the SHRs compared to the Wistar rats. Administration of both drugs decreased the WT/ID ratio in all of the groups, except for the prazosin-treated SHR group (Fig. 3D).

The circumferential tension of the carotid artery in the Wistar rats and the SHR did not significantly differ. After the administration of both drugs to the Wistar rats, the circumferential tension of the carotid artery was increased. A similar increase was observed in the losartan-treated SHRs compared to the untreated SHRs. In contrast, the administration of prazosin to the SHRs did not change the value of the circumferential tension of the carotid artery (Fig. 4). 


\section{Integrated responses}

Acute i.v. application of increasing doses of acetylcholine $(0.1,1$ and $10 \mu \mathrm{g})$ induced similar integrated responses in the cardiovascular system in the SHRs and the Wistar rats. In the groups of Wistar rats treated with prazosin and losartan, acetylcholine did not induce significant changes in BP decrease, except at the highest dose $(10 \mu \mathrm{g})$ in the prazosin group. Administration of individual doses of acetylcholine to the SHRs treated with prazosin and losartan, significantly improved the acetylcholine-induced decrease in the systemic BP (Fig. 5).

\section{Discussion}

During the $4^{\text {th }}$ week after birth (when the experiments were started), the BP did not differ significantly between the Wistar rats and the SHRs. From the $4^{\text {th }}$ week to the $9^{\text {th }}$ week of age, the BP values continued to increase in both

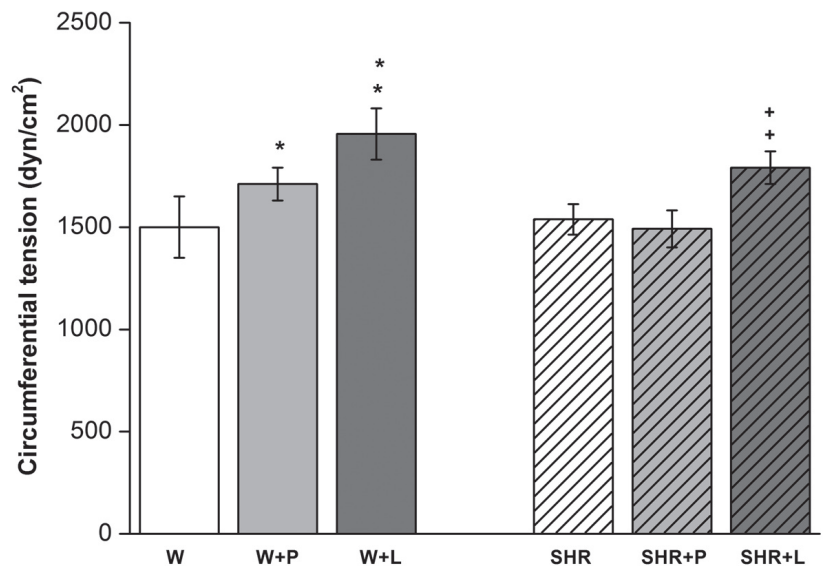

Figure 4. Circumferential tension in carotid artery of control Wistar rats, Wistar rats treated with prazosin, Wistar rats treated with losartan, spontaneously hypertensive rats, SHR receiving prazosin, and SHR receiving losartan. The values represent the data at the end of the experiment. Data are means \pm S.E.M. ${ }^{\star} p<0.05,{ }^{* *} p<0.01 v$ s. Wistar rats; ${ }^{++} p<0.01$ vs. SHR. For abbreviations see Fig. 1.
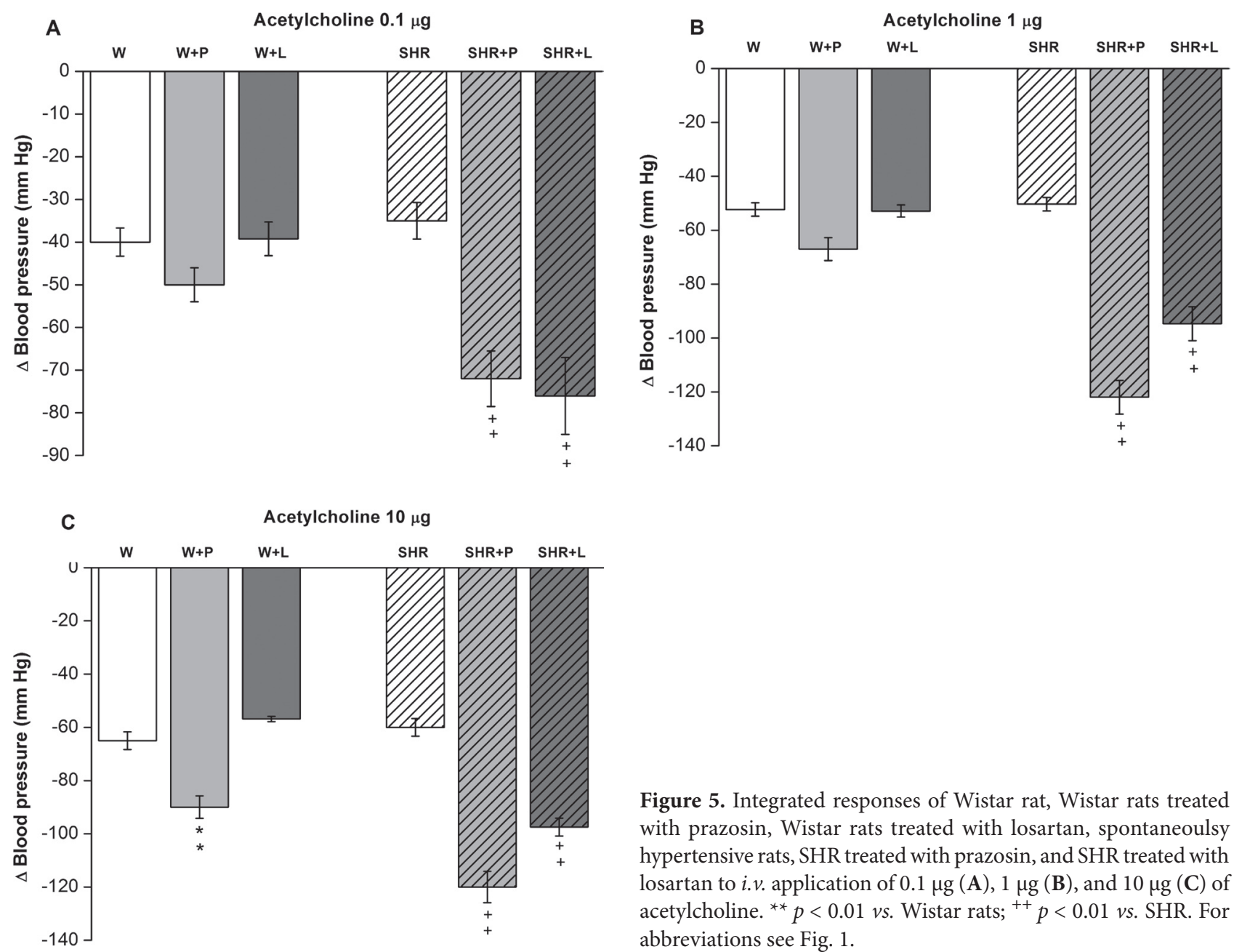

Figure 5. Integrated responses of Wistar rat, Wistar rats treated with prazosin, Wistar rats treated with losartan, spontaneoulsy hypertensive rats, SHR treated with prazosin, and SHR treated with losartan to i.v. application of $0.1 \mu \mathrm{g}(\mathbf{A}), 1 \mu \mathrm{g}(\mathbf{B})$, and $10 \mu \mathrm{g}(\mathbf{C})$ of acetylcholine. ${ }^{* *} p<0.01 v s$. Wistar rats; ${ }^{++} p<0.01 v s$. SHR. For abbreviations see Fig. 1 . 
groups; however, the increase was more rapid in the SHRs. During the $5^{\text {th }}$ week after birth, BP was already significantly increased and at the end of the experiment, it was increased by approximately $37 \%$. This BP increase was accompanied by hypertrophy of the heart and arterial wall. Hypertrophy of the heart (as indicated by the HW/BW ratio) during hypertension is an adaptive cardiac response to mechanical overload. The thickening of arterial media reflects the adaptation of the arterial smooth muscle cells to increased BP (primarily systolic), although humoral factors may also be involved (Owens 1995). Our data are in good agreement with the results of several experiments that document that early ontogeny represents the most active period, during which pathological alterations in the cardiovascular system of SHRs occur.

The long-term administration of prazosin and losartan to the SHRs produced a decrease in the BP and trophicity of the heart. Both of these effects are supposed to be beneficial outcomes of drug treatments on the cardiovascular system during hypertension. Nevertheless, the drugs were unable to prevent the increase in the BP completely, and at the end of the experiments, the BP was still significantly increased in the SHRs compared to the Wistar rats. In contrast to the $\mathrm{BP}$ trends, both of these drugs completely prevented the hypertrophy of the heart in the SHRs (HW/BW did not differ from that observed in the Wistar rats). The question remains whether BP lowering is a necessary condition for this regression. There are several lines of evidence that suggest that there is a positive correlation between BP and trophicity of the heart and arterial wall, although there also exist data that question this hypothesis. Griffin et al. (1991) reported that infusion of subpressor doses of ang II leads to an increase in the thickness of the arterial media and ratio of media to lumen in the resistant arteries. Further, the discrepancy between BP and trophicity of the arterial wall has also been observed in 4-week-old Wistar rats that were progenies of NO-deficient parents, where hypertension was accompanied with hypotrophy of the heart and arterial wall (Kristek and Gerova 2004). In addition, BPindependent decrease in cardiac hypertrophy was observed after long-term administration of exogenous NO donors and/or sildenafil to SHRs (Kristek et al. 2007). The relative independence between BP and trophicity of the heart and arterial wall is supported by experiments that demonstrated that long-term control of $\mathrm{BP}$ with atenolol did not reverse the hypertrophic process observed in essential hypertension (Schiffrin et al. 1994). A more pronounced effect of prazosin and losartan on cardiac trophicity compared to BP raises the question whether imbalance between trophicity and BP is a real beneficial effect. It should be stressed that the decreased mass of the myocardium has to surmount relatively higher peripheral resistance, which could later lead to cardiac insufficiency.
The administration of prazosin and losartan to Wistar rats produced a decrease in WT, CSA, and WT/ID ratio with a small effect on ID. In SHRs, the administration of losartan had an effect on the arterial wall that was similar to that observed in Wistar rats; however, there was no effect of prazosin administration on the arterial wall. Similar effect of losartan treatment has been observed by Sabri et al. (1997). In ang II-induced hypertensive rats, losartan elicited a decrease in the media thickness of aorta and coronary artery. On the other hand, long-term administration of losartan to young SHRs produced a decrease in BP and increase in the ID, without affecting arterial wall hypertrophy, of mesenteric resistant vessels (Rizzoni et al. 1998). According to Laplace's law, a mutual relation between BP, ID and arterial WT determines the circumferential stress in arterial walls. Interrelationship among these parameters guarantees an appropriate adaptive mechanism in the arteries to respond to altered conditions. Due to this, the circumferential stress is similar in the arteries of SHRs and normotensive Wistar rats (Cunha et al. 1997; Kristek et al. 2007). The greater antiproliferative effects of prazosin (in Wistar rats) and losartan (in Wistar rats and SHRs) on the arterial wall in contrast to their antihypertensive effect disturbs the delicate balance between the BP, ID and WT. This disturbance results in an increase in the circumferential stress, which influences the functional parameters of the vascular tree. Thubrikar and Roubicsek (1995) showed that a decrease in circumferential stress leads to a decrease in the pathological changes in the arterial wall, and an increase circumferential stress could trigger the opposite effects. In addition, increased circumferential stress in the coronary artery was observed after losartan administration in SHRs (Koprdova et al. 2009).

In young SHRs, the activity of ang II and the SNS is increased compared to normotensive rats (Head 1989). Experiments have demonstrated that the effect of alfa adrenoceptor inhibition is time dependent. When the alfal adrenoceptor antagonist, terazosin, was administered to SHRs during their $3^{\text {rd }}$ postnatal week, it prevented the increase in BP when the SHRs became adults (McCarty et al. 1987). When administration of the same drug was delayed till the $4^{\text {th }}$ postnatal week, the increase in BP was not affected (Jonsson et al. 1992). In comparison with the experiment described by McCarty et al. (1987), the lower decrease in BP observed in our experiment after prazosin administration could be associated with the time that the administration was initiated ( $4^{\text {th }}$ week). A more pronounced effect, i.e., normalization of peripheral resistance and prevention of cardiac hypertrophy, can be achieved when inhibition of $\alpha 1$ adrenoceptors using prazosin is accompanied by sympathectomy (Korner et al. 1993).

The higher effect of losartan compared to prazosin on hypertrophy of the heart and arterial wall supports the suggestion that ang II is a strong proliferative agent that 
is directly involved in the development of cardiovascular hypertrophy (Griendling and Alexander, 1995). In comparison with our data, a more pronounced effect of losartan administration was observed by Soltis et al. (1993) and Kaneko et al. (1996) when they used a higher dose of losartan $(30 \mathrm{mg} / \mathrm{kg} /$ day) and a different mode of application (subcutaneous).

We observed similar effects of losartan administration on the heart and arterial wall of Wistar rats compared to SHRs. In contrast to our results, Sabri et al. (1997) did not observe any effect on the $\mathrm{BP}$ and $\mathrm{HW} / \mathrm{BW}$ ratio in normotensive Wistar rats after losartan administration. Moreover, they found hypertrophy of the aorta and coronary artery wall. They used the same dose of losartan $(10 \mathrm{mg} / \mathrm{kg} /$ day $)$, which was administered by daily gavage. Our experiments started when the animals were 4 weeks old (weighed approximately $250 \mathrm{~g}$ ), and it lasted for 6 weeks. In the experiment of Sabri et al. (1997), the animals weighed $300 \mathrm{~g}$ (the age of the animals was not mentioned), and the duration of the experiment was 3 weeks. Considering the weight of the rats, we hypothesize that those animals were older than ours. Furthermore, we speculate that this variability could be due to a different type of artery than the one we used in our experiments. However, these hypotheses can only partially explain the differences between our results.

In the Wistar rats and the SHRs, acetylcholine (at all doses used) induced similar responses with regards to systemic BP. This result could be associated with satisfactory NO production by the endothelium in the SHRs. The suggestion is supported with our previous experiment in which a longterm increase in the NO level due to the administration of NO donors did not influence the BP and geometry of the conduit arteries (Kristek et al. 2003). Nevertheless, there is no consensus in the literature as to whether in SHRs, NO level and acetylcholine-induced relaxation is increased or decreased in comparison with Wistar rats. In the Wistar rats in the present study, acetylcholine-induced a decrease in the BP only after the highest dose of acetylcholine was administered to the prazosin treated rats (the absolute values were increased in all doses of acetylcholine; however, they were not significant). In the SHRs, the acetylcholine treatment induced a significant decrease in the systemic BP in the prazosin and losartan groups. Further, relaxation in response to acetylcholine was observed by Schiffrin et al. (1994) using pressurized precontracted mesenteric arteries isolated from losartan-treated SHRs. They suggested that the improvement might be due to the lowering of BP. AT1 receptor subtypes are present in the endothelium of arteries, and their inhibition could result in the improvement of endothelial functions. Schiffrin et al. (1994) supposed that the improvement could additionally result from the action on AT2 receptors, which are not blocked by losartan. In SHRs, increases in oxidative stress have been described after alfa1 adrenoceptor stimulation (Bleeke et al. 2004). Superoxides decrease the availability of NO from eNOS. Reduction of superoxide concentration lowers the BP in SHRs (Vaziri et al. 2000). Prazosin decreases NAD(P)Hdependent production of superoxide radicals induced by cocaine (Isabelle et al., 2005). Ang II, via AT1 receptors, appears to be responsible for the activation of $\mathrm{NAD}(\mathrm{P}) \mathrm{H}$ oxidase (main contributor to oxidative stress in the vessel wall) in SHRs (Welch and Wilcox 2001; Zalba et al. 2001). We suggest that inhibition of alfa1 and AT1 receptors improves the bioavailability of $\mathrm{NO}$ and could be responsible for the higher responses of the cardiovascular system to acetylcholine administration. Improvement in acetylcholine-induced BP decrease in SHRs treated with prazosin and/or losartan is in accordance with in vitro experiments. In those experiments, significant improvement in the concentration-response curves for relaxation induced by acetylcholine in aortic rings from SHRs was observed by Soltis (1993) and Torok et al. $(2005,2006)$.

In conclusion, the BP of 4-week-old SHRs did not differ from age-matched Wistar rats. In the SHRs, the BP rapidly increased from the $5^{\text {th }}$ to the $9^{\text {th }}$ postnatal week. Long-term administration of prazosin and losartan partially prevented the elevation in BP and improved the trophicity of the heart in both normotensive Wistar rats and SHRs. Losartan and prazosin decreased trophicity of the arterial wall in Wistar rats while in SHR losartan only. Both drugs significantly improved the acetylcholine-induced decrease in the BP in the SHRs; however, this effect was not observed in the Wistar rats.

Acknowledgement. The study was supported by research grant VEGA 2/0019/12, VEGA 2/0111/12 and grant 2012/51-SAV-1. The authors would like to thank L. Kosnacova for technical assistance.

\section{References}

Adams M. A., Bobik A., Korner P. I. (1989): Differential development of vascular and cardiac hypertrophy in genetic hypertension. Relation to sympathetic function. Hypertension 14, 191-202 http://dx.doi.org/10.1161/01.HYP.14.2.191

Bleeke T., Zhang H., Madamanchi N., Patterson C., Faber J. E. (2004): Catecholamine-induced vascular wall growth is dependent on generation of reactive oxygen species. Circ. Res. 94, 37-45 http://dx.doi.org/10.1161/01.RES.0000109412.80157.7D

Cebova M., Kristek F. (2011): Age-dependent ultrastructural changes of coronary artery in spontaneously hypertensive rats. Gen. Physiol. Biophys. 30, 364-372 http://dx.doi.org/10.4149/gpb_2011_04_364

Clubb F. J. Jr., Bell P. D., Kriseman J. D., Bishop S. P. (1987): Myocardial cell growth and blood pressure development in neonatal spontaneously hypertensive rats. Lab. Invest. 56, 189-197 
Crawford D. C., Chobanian A. V., Brecher P. (1994): Angiotensin II induces fibronectin expression associated with cardiac fibrosis in the rat. Circ. Res. 74, 727-739 http://dx.doi.org/10.1161/01.RES.74.4.727

Cunha R. S., Dabire H., Bezie I., Weiss A. M., Chanouche-Terzara K., Laurent S., Safar M. E., Lacolley P. (1997): Mechanical stress of the carotid artery at the early phase of spontaneous hypertension in rats. Hypertension 29, 992-998 http://dx.doi.org/10.1161/01.HYP.29.4.992

Gray S. D. (1984): Pressure profiles in neonatal spontaneously hypertensive rats. Biol. Neonate 45, 25-32 http://dx.doi.org/10.1159/000241759

Griendling K. K., Alexander R. W. (1995): Angiotensin, other pressors and the transduction vascular smooth muscle contraction. In Hypertension, Pathophysiology, Diagnosis, and Management (Ed. B. Laragh), pp. 524-541, Raven Press, New York

Griffin S. A., Brown W. C. B., MacPherson F., McGrath J. C., Wilson V. G., Korsgaard N., Mulvany M. J., Lever A. F. (1991): Angiotensin II causes vascular hypertrophy in part by a nonpressor mechanism. Hypertension 17, 626-635 http://dx.doi.org/10.1161/01.HYP.17.5.626

Head R. J. (1989): Hypernoradrenergic innervation: its relationship to functional and hyperplastic changes in the vasculature of the spontaneously hypertensive rat. Blood Vessels 26, 1-20

Isabelle M., Monteil C., Moritz F., Dautreaux B., Henry J. P., Richard V., Mulder P., Thuillez C. (2005): Role of a1-adrenoreceptors in cocaine-induced NADPH oxidase expression and cardiac dysfunction. Cardiovasc. Res. 67, 699-704 http://dx.doi.org/10.1016/j.cardiores.2005.04.026

Jonsson J. R., Head R. J., Frewin D. B. (1992): Effect of 1-adrenoceptor blockade on the development of hypertension in the spontaneously hypertensive rat. Eur. J. Pharmacol. 211, $263-268$ http://dx.doi.org/10.1016/0014-2999(92)90538-F

Kaneko K., Susic D., Nunez E., Frohlich E. D. (1996): Losartan reduces cardiac mass and improves coronary flow reserve in the spontaneously hypertensive rat. J. Hypertens. 14, 645-653 http://dx.doi.org/10.1097/00004872-199605000-00015

Koprdova R., Cebova M., Kristek F. (2009): Long-term effects of losartan administration on the cardiovascular system of young SHR. Physiol. Res. 58, 327-335

Korner P., Bobik A., Oddie C., Friberg P. (1993): Sympathoadrenal system is critical for structural changes in genetic hypertension. Hypertension 22, 243-252

http://dx.doi.org/10.1161/01.HYP.22.2.243

Kristek F. (2000): Pentaerythrityl tetranitrate attenuates structural changes in conduit arteries evoked by long-term NO-synthase inhibition. Br. J. Pharmacol. 130, 450-456 http://dx.doi.org/10.1038/sj.bjp.0703307

Kristek F., Faberova V., Varga I. (2003): Long-term effect of molsidomine and pentaerythrityl tetranitrate on cardiovascular system of spontaneously hypertensive rats. Physiol. Res. 52, 709-717

Kristek F., Gerova M. (2004): Hypotrophy of conduit artery walls of the offspring of nitric oxide-defective rats. Braz. J. Med. Biol. Res. 37, 601-606
http://dx.doi.org/10.1590/S0100-879X2004000400018

Kristek F., Koprdova R., Cebova M. (2007): Long-term effects of early administered sildenafil and NO donor on the cardiovascular system of SHR. J. Physiol. Pharmacol. 58, 33-43

Kristek F., Koprdova R. (2011): Long-term effect of prazosin administration on blood pressure, heart and structure of coronary artery of young spontaneously hypertensive rats. J. Physiol. Pharmacol. 62, 295-301

McCarty R., Kirby R. F., Cierpial M. A., Jenal T. J. (1987): Accelerated development of cardiac sympathetic responses in spontaneously hypertensive (SHR) rats. Behav. Neural Biol. 48, 321-333 http://dx.doi.org/10.1016/S0163-1047(87)90879-X

Okamoto K., Aoki K. (1963): Development of a strain of spontaneously hypertensive rats. Jpn. Circ. J. 27, 282-293 http://dx.doi.org/10.1253/jcj.27.282

Owens G. K. (1995): Regulation of differentiation of vascular smooth muscle cells. Physiol. Rev. 75, 487-517

Rizzoni D., Porteri E., Piccoli A., Castellano M., Bettoni G., Muiesan M. L., Pasini G., Guelfi D., Mulvany M. J., Rosei E. A. (1998): Effects of losartan and enalapril on small artery structure in hypertensive rats. Hypertension. 32, 305-310 http://dx.doi.org/10.1161/01.HYP.32.2.305

Ruiz-Gayo M., Somoza B., Bravo R., Fernandez-Alfonso M. S., Gonzalez C. (2000): Chronic losartan treatment decreases angiotensin II-mediated facilitation of noradrenaline release in the caudal artery of spontaneously hypertensive rats. Life Sci. 67, 3153-3162 http://dx.doi.org/10.1016/S0024-3205(00)00900-0

Sabri A., Levy B., Poitevin P., Caputo L., Faggin E., Marotte F., Rappaport L., Samuel J. L. (1997): Differential role of AT1 and AT2 receptors subtypes in vascular trophic and phenotypic changes in response to a stimulation with angiotenzín II. Arterioscler Tromb. 17, 257-264 http://dx.doi.org/10.1161/01.ATV.17.2.257

Schiffrin E. L., Deng L. Y., Larochelle P. (1994): Effects of a $\beta$-blocker or a converting enzyme inhibitor on resistance arteries in essential hypertension. Hypertension 23, 83-91 http://dx.doi.org/10.1161/01.HYP.23.1.83

Soltis E. E. (1993): Alterations in vascular structure and funetion after short-term losartan treatment in spontaneously hypertensive rats. J. Pharmacol Exp. Ther. 266, 642-646

Soltis E. E., Jewell A. L., Dwoskin L. P., Cassis L. A. (1993): Acute and chronic effects of losartan (DUP-753) on blood-pressure and vascular reactivity in normotensive rats. Clin Exp Hypertens. 15, 171-184 http://dx.doi.org/10.3109/10641969309041618

Thubrikar M. J., Robicsek F. (1995): Pressure-induced arterial-wall stress and atherosclerosis. Ann. Thorac. Surg. 59, 1594-1603 http://dx.doi.org/10.1016/0003-4975(94)01037-D

Torok J., Koprdova R., Cebova M., Kristek F. (2005): Effect of losartan on systolic blood pressure and endothelial function in young SHR. Cardiol. 14, K/C106-107

Torok J., Koprdova R., Cebova M., Kristek F. (2006): Effect of prazosin on functional and morphological cardiovascular parameters in young spontaneously hypertensive rats. Physiol. Res. 55, 11 
Vaziri N. D., Ni Z., Oveisi F., Trnavsky-Hobbs D. L. (2000) Effect of antioxidant therapy on blood pressure and NO synthase expression in hypertensive rats. Hypertension 36, 957-964

http://dx.doi.org/10.1161/01.HYP.36.6.957

Welch W. J., Wilcox C. S. (2001): AT1 receptor antagonist combats oxidative stress and restores nitric oxide signaling in the SHR. Kidney Int. 59, 1257-1263

http://dx.doi.org/10.1046/j.1523-1755.2001.0590041257.x
Zalba G., Beaumont F. J., San Jose G., Fortuno A., Fortuno M. A., Diez J. (2001): Is the balance between nitric oxide and superoxide altered in spontaneously hypertensive rats with endothelial dysfunction? Nephrol Dial Transplant. 16 (Suppl 1), 2-5

http://dx.doi.org/10.1093/ndt/16.suppl_1.2

Received: October 16, 2012

Final version accepted: December 19, 2012 